\title{
Histopathological efficiency of amifostine in radiation-induced heart disease in rats
}

\author{
Gurses $\mathrm{I}^{1}$, Ozeren $\mathrm{M}^{2}$, Serin $\mathrm{M}^{3}$, Yucel $\mathrm{N}^{4}$, Erkal $\mathrm{HS}^{5}$ \\ Mersin University Medical Faculty, Department of Pathology, Mersin, Turkey. \\ iclalgurses@gmail.com
}

\begin{abstract}
OBJECTIVE: Amifositine is a phosphorylated thiol that holds its radioprotective actions by several indirect mechanisms. The purpose of this study was to evaluate histopathologically whether amifositine administration prior to irradiation would have a long-term protective effect on heart tissue in an experimental rat model.

METHODS: Single dose of 18 Gy radiation and sham radiation exposure were used in related groups. A dose of $200 \mathrm{mg} / \mathrm{kg}$ of amifostine was injected intraperitoneally $30 \mathrm{~min}$ prior to radiation exposure. Analyses were performed 6 months after irradiation.

RESULTS: Vascular damage and vasculitis were significantly decreased in amifositine treatment group. At the same time, significant thickening of the medial layer was accompanied by vascular damage in irradiated groups. The number and severity of myocyte necrosis were diminished with amifostine.

Nevertheless, it could not prevent epicardial and myocardial fibrosis. Severe myocardial fibrosis was observed prominently in three regions, particularly on the apex, tips of papillary muscles and in sites adjacent to the atrioventricular valves. The anti-inflammatory effect of amifostine was not seen.

CONCLUSION: The development of vascular damage and vasculitis were prevented by the use of amifostine. There was a correlation between vascular damage and fibrosis development. According to histopathological results, amifostine could be used as a protective agent against the side effects of radiotherapy (Tab. 4, Fig. 2, Ref. 22). Text in PDF www.elis.sk.

KEY WORDS: amifostine, histopathology, irradiation, radiation-induced heart disease.
\end{abstract}

\section{Introduction}

Radiotherapy (RT) has advanced and become one of the important treatment modalities for various types of cancers. Nowadays, half of all cancer patients receive RT and many of them are successfully treated (1). These cancer survivors return to their normal lives but normal tissue toxicity of RT is becoming a bigger concern over long period of time. Recent data from several independent reports have provided substantial evidence that mean heart doses of $\leq 20$ Gy and even $\leq 5$ Gy can increase the risk of cardiac damage (2). Although modern RT techniques have reduced radiation exposure of the heart, they may not reduce the cardiotoxicity and it appears that these methods may still give rise to cardiovascular

${ }^{1}$ Mersin University Medical Faculty, Department of Pathology, Mersin, Turkey, ${ }^{2}$ Mersin University Medical Faculty, Department of Cardiovascular Surgery, Mersin, Turkey, ${ }^{3}$ Acibadem University Adana Hospital, Department of Radiation Oncology, Adana, Turkey, ${ }^{4}$ Inönü University Medical Faculty, Department of Emergency Medicine, Malatya, Turkey, and ${ }^{5} \mathrm{Sa}-$ karya University Medical Faculty, Department of Radiation Oncology, Sakarya, Turkey

Address for correspondence: I. Gurses, Mersin University Medical Faculty, Department of Pathology, 34. Cadde Ciftlikköy kampüsü, 33343 Mersin, Turkey.

Phone: +90.324.2410000/0532 5792181 disease (3). Heart damage caused by RT is called radiation-induced heart disease (RIHD). The diagnosis of RIHD is quite difficult and it can be acute, subacute or chronic. RIHD manifests as pericarditis (both acute and chronic forms), coronary artery disease (accelerated atherosclerosis), conduction abnormalities, valvulitis, myocarditis and notably, pericardial and myocardial fibrosis. The disease is progressive; therefore, the manifestations may become clinically apparent several years after irradiation (4).

The inactive prodrug amifostine (WR-2721), a member of sulfhydryl-containing class of agents (a phosphorylated thiol), is converted to its active form, WR-1065, by alkaline phosphatase of vascular endothelial cells induced dephosphorylation. Amifostine holds its radioprotective actions by several indirect mechanisms, particularly it scavenges radiation-induced free radicals, repairs the chemical damage by donating hydrogen atoms, and induces intracellular hypoxia by auto-oxidation (5). Additionally, amifostine may also act as a radioprotector by its direct actions on cells by arresting the cell cycle at $\mathrm{G} 1$ phase and inducing $\mathrm{p} 21$ expression (6). There are few articles in literature investigating the protective effect of amifostine on RIHD $(7,8)$.

The purpose of this study was to evaluate histopathologically whether amifositine administration prior to irradiation would have a long-term protective effect on heart tissue in an experimental rat model. 


\section{Materials and methods}

\section{Animal model}

All experimental procedures and protocols used in this investigation were reviewed and approved by the "Animal Ethics Committee of Inonu University" following the approval of the study design. Study was designed as a late-stage study comprising a time period of 6 months (9).

Forty male Wistar rats (aged between 10 and 12 weeks with body weights between 200 and 250 g) were randomly divided into four groups. The number of rats in each group was ten. Each group was kept in separate cages in rooms with controlled light and temperature adjustments and was fed with standard chow and water ad libitum. Group 1 received no amifositine and underwent radiation exposure. The rats in group 2 received amifositine and underwent radiation exposure. The rats in group 3 received amifositine and underwent sham radiation. The rats in group 4 received no amifositine and underwent sham radiation.

\section{Amifostine}

The rats in group 2 and 3 were given amifostine $200 \mathrm{mg} / \mathrm{kg}$ (Ethyol, Medimmune Pharma B.V. the Netherlands) intraperitoneally $30 \mathrm{~min}$ prior to being exposed to radiation. Sodium chloride $0.9 \%$ was prepared at an equal volume with amifostine and the rest of the procedure was applied identically to rats in groups 1 and 4 .

\section{Radiation Exposure}

Prior to radiation exposure or sham radiation, the rats received anesthesia using ketamine (Ketalar, Pfizer Ilaclari Limited Sirketi, Istanbul, Turkey) at a dose of $80 \mathrm{mg} / \mathrm{kg}$ and xylazine (Rompun, Bayer Türk Kimya Sanayi Limited Sirketi, Istanbul, Turkey) at a dose of $5 \mathrm{mg} / \mathrm{kg}$ via an intraperitoneal injection. The rats were immobilized in supine position on a rough surface by taping the extremities. In groups 1 and 2, radiation was delivered in the supine position on a Cobalt-60 unit using an anterior field in size of 2.5 x $2.5 \mathrm{~cm}^{2}$ and a single fraction of 18 Gy defined for a depth of 2.5 $\mathrm{cm}$ through an anterior portal. The rest of the body was shielded with lead plates. In group 3, sham radiation was delivered on a Cobalt-60 unit over the same fraction duration. Following radiation exposure or sham radiation, the animals were closely observed until recovery from anesthesia.

\section{Euthanasia}

The rats were euthanized 6 months following radiation exposure or sham radiation. Prior to euthanasia, the rats received anesthesia with propofol (Propofol, Abbott Laboratuvari Anonim Sirketi, Istanbul, Turkey) at a dose of $50 \mathrm{mg} / \mathrm{kg}$ administered via an intraperitoneal injection. Euthanasia was performed by transcardiac perfusion of $0.9 \%$ sodium chloride.

\section{The histopathologic evaluation}

Hearts were dissected rapidly from the mediastinum and kept in $10 \%$ buffered formaldehyde for 24 hours. Longitudinal tissue slices of 3-4 mm in thickness on average showing the four chambers of the heart were taken after following the routine tis- sue processing. Two different levels of section ( $4 \mu \mathrm{m}$ thick) were obtained. Sections were stained using hematoxylin and eosin for general tissue characterization. Total collagen accumulation was determined by preparing tissue sections with Masson's trichrome stain. The blinded histopathological evaluation was performed under the light microscope (Olympus BX51, Japan) using a semiquantitative scoring system for the severity and extent of histological parameters, in which left and right ventricles were examined separately according to the three layers of the heart (endocardium, myocardium, epicardium).

Inflammation, thrombus, fibrosis, myocyte necrosis and vascular damage were also the items used to describe radiation injury to the ventricles. The degree of inflammation for each of layers of the ventricles was scaled from 0 to 3 as (0) no inflammation, (1) mild, (2) moderate or (3) severe inflammation. Thrombus was defined as existent (1) or absent (0). Myocyte necrosis was graded as (0) no necrosis, (1) single cell necrosis, (2) more than one cell. Fibrosis in the myocardium of ventricles was quantified by a graded scale from 0 to 4 as follows: (0) no fibrosis, (1) one small area affected, (2) area of less than $5 \%$ affected, (3) area of $5-10 \%$ affected or (4) over $10 \%$ affected (7). Mesh configuration was defined as the presence of the web form of fibrosis covering each myofibril and was described as present or absent. Fibrosis of endocardial and epicardial layers were defined as existent (1) or absent (0). Additionally, vascular damage in the myocardium of ventricles was scored according to a graded scale from 0 to 3 as follows: (0) no fibrosis while the thickness of adventitia is up to $50 \%$ of the media, (1) mild fibrosis while adventitia = media, (2) moderate fibrosis with thickness of adventitia being greater than twofold of the media, (3) severe fibrosis with thickness of adventitia being greater than threefold of the media (7).

\section{Statistical analysis}

Median item scores for fibrosis, presence of mesh configuration, thrombus, inflammation, vascular damage and necrosis were compared using the Mann-Whitney U test. Correlation between the parameters was determined using the Bi Variate Correlation (Pierson) test $(\mathrm{r}=$ correlation coefficient). Statistical significance was defined as the $p$ value being less than or equal to 0.05 . Number of items related with the parameters was given as numbers (n) in brackets.

\section{Results}

\section{Histopathologic evaluation Chronic inflammation}

Myocardial inflammation in all groups is presented in Table 1. No significant difference was found between groups 1 and 2 which we expected owing to the protective effect of amifositine. Statistical differences were found between the RT groups and controls but it has no importance in terms of protection. Epicardial inflammation showed results similar to those of myocardial inflammation (Tab. 2). Histopathologically, pronounced inflammation in both ventricles was found in groups 1 and 2 compared to that in ventricles in groups 3 and 4 . 
Tab. 1. Distribution of myocardial inflammation in all groups.

\begin{tabular}{ccccccc}
\hline & & \multicolumn{4}{l}{ Myocardial inflammation scores } & \multirow{2}{*}{ Significant $\mathrm{p}$ values } \\
\cline { 2 - 5 } & 0 & 1 & 2 & 3 & \\
\hline \multirow{3}{*}{$\mathrm{RV}$} & 1 & 0 & 5 & 5 & 3 & $0.03(\mathrm{G} 1-3)$ \\
& 2 & 0 & 4 & 4 & 2 & $0.02(\mathrm{G} 2-3)$ \\
& 3 & 0 & 0 & 0 & 0 & \\
& 4 & 0 & 0 & 0 & 0 & $0.01(\mathrm{G} 1-4)$ \\
\hline \multirow{2}{*}{$\mathrm{LV}$} & 1 & 0 & 5 & 3 & 2 & $0.03(\mathrm{G} 1-3)$ \\
& 2 & 0 & 4 & 3 & 2 & $0.04(\mathrm{G} 2-3)$ \\
& 3 & 0 & 0 & 0 & 0 & \\
& 4 & 0 & 0 & 0 & 0 & $0.02(\mathrm{G} 1-4)$ \\
\hline
\end{tabular}

$\mathrm{RV}$ - right ventricle, $\mathrm{LV}$ - left ventricle; bracket indicates group differences

Tab. 2. Distribution of epicardial inflammation in all groups.

\begin{tabular}{ccccccc}
\hline & & \multicolumn{6}{c}{ Epicardial inflammation scores } & \multirow{2}{*}{ Significant p values } \\
\cline { 2 - 6 } & & 0 & 1 & 2 & 3 & \\
\hline \multirow{4}{*}{$\mathrm{RV}$} & 1 & 0 & 7 & 6 & 1 & $0.04(\mathrm{G} 1-3)$ \\
& 2 & 0 & 3 & 3 & 2 & $0.03(\mathrm{G} 2-3)$ \\
& 3 & 0 & 0 & 0 & 0 & \\
& 4 & 0 & 0 & 0 & 0 & $0.01(\mathrm{G} 1-4)$ \\
\hline \multirow{4}{*}{$\mathrm{LV}$} & 1 & 0 & 2 & 1 & 0 & \\
& 2 & 0 & 2 & 1 & 1 & $0.045(\mathrm{G} 2-3)$ \\
& 3 & 0 & 0 & 0 & 0 & \\
& 4 & 0 & 0 & 0 & 0 & \\
\hline
\end{tabular}

RV - right ventricle, $\mathrm{LV}$ - left ventricle

Tab. 3. Distribution of vascular damage in all group.

\begin{tabular}{|c|c|c|c|c|c|c|}
\hline & \multirow{2}{*}{ Groups } & \multicolumn{4}{|c|}{ Grades of vascular damage } & \multirow{2}{*}{ Significant $\mathrm{p}$ values } \\
\hline & & 0 & 1 & 2 & 3 & \\
\hline \multirow{4}{*}{ RV } & 1 & 0 & 8 & 0 & 2 & \\
\hline & 2 & 0 & 4 & 0 & 0 & $0.011(\mathrm{G} 1-\mathrm{G} 2)$ \\
\hline & 3 & 0 & 0 & 0 & 0 & $0.003(\mathrm{G} 1-\mathrm{G} 3)$ \\
\hline & 4 & 0 & 0 & 0 & 0 & $0.001(\mathrm{G} 1-\mathrm{G} 4)$ \\
\hline \multirow{4}{*}{ LV } & 1 & 0 & 3 & 0 & 0 & \\
\hline & 2 & 0 & 4 & 0 & 0 & $\mathrm{NS}(\mathrm{G} 1-\mathrm{G} 2)$ \\
\hline & 3 & 0 & 0 & 0 & 0 & $0.04(\mathrm{G} 1-\mathrm{G} 3)$ \\
\hline & 4 & 0 & 0 & 0 & 0 & 0.03 (G1-G4) \\
\hline
\end{tabular}

Tab. 4. Distribution of myocardial fibrosis in all groups.

\begin{tabular}{|c|c|c|c|c|c|c|c|}
\hline & \multirow{2}{*}{ Groups } & \multicolumn{5}{|c|}{ Grades of myocardial fibrosis } & \multirow{2}{*}{ Significant $\mathrm{p}$ values } \\
\hline & & 0 & 1 & 2 & 3 & 4 & \\
\hline \multirow{4}{*}{ RV } & 1 & 0 & 1 & 4 & 2 & 2 & \\
\hline & 2 & 0 & 1 & 6 & 2 & 0 & NS (G1-G2) \\
\hline & 3 & 0 & 0 & 0 & 0 & 0 & $0.01(\mathrm{G} 1-\mathrm{G} 3)$ \\
\hline & 4 & 0 & 0 & 0 & 0 & 0 & $0.001(\mathrm{G} 2-\mathrm{G} 4)$ \\
\hline \multirow{4}{*}{ LV } & 1 & 0 & 5 & 2 & 2 & 0 & $0.001(\mathrm{G} 1-\mathrm{G} 4)$ \\
\hline & 2 & 0 & 3 & 3 & 2 & 0 & NS (G1-G2) \\
\hline & 3 & 0 & 0 & 0 & 0 & 0 & $0.045(\mathrm{G} 2-\mathrm{G} 3)$ \\
\hline & 4 & 0 & 0 & 0 & 0 & 0 & 0.001 (G2-G4) \\
\hline
\end{tabular}

\section{Vascular damage}

While normal vascular structures were existent in groups 3 and 4 (Fig. 1A,B), vascular damage was seen in groups 1 and 2 which were exposed to radiation (Figs 1C,D,E,F, 2A,B). We found that right ventricles were more affected in group 1 according to the vascular damage scoring. In group 2, right and left ventricles were equally affected (Tab. 3). Statistically significant

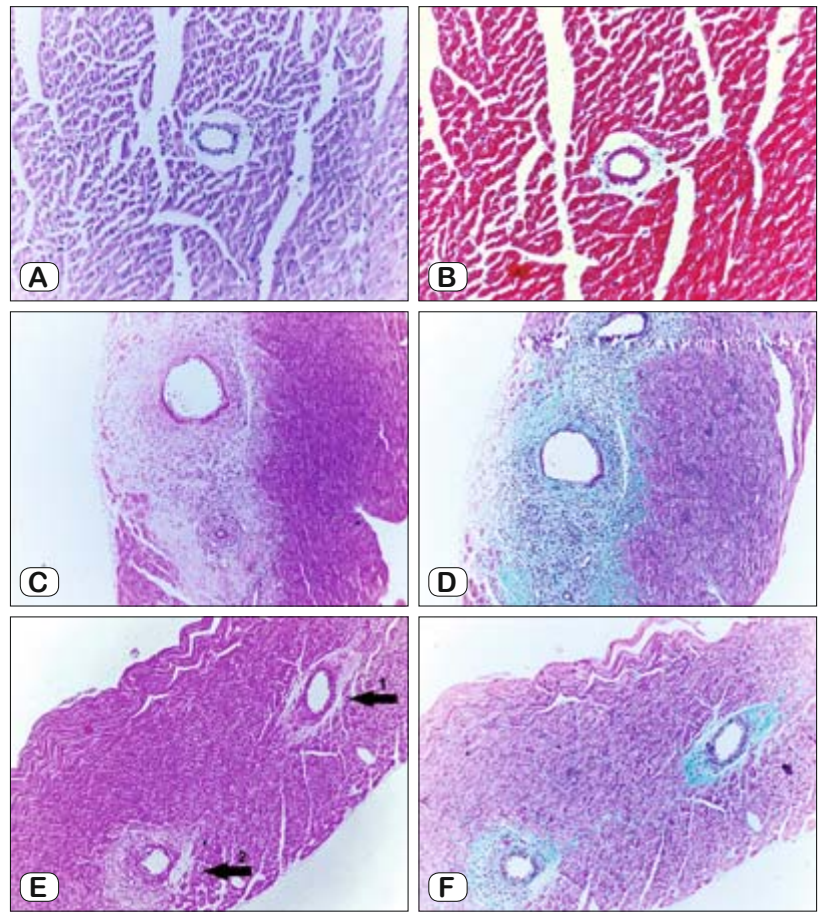

Fig. 1. A-B: Normal vascular and myocardial appearance (A: $H \& E$ x200, B: Masson's trichrome staining x200), C-D: Vascular damage and vasculitis characterized by inflammation and fibrinoid necrosis in the vessel wall of radiation group (Group 1). (C: H\&E, x40; D: Masson's trichrome staining, x40), E-F: Diminished vascular damage (Arrow 1) and vasculitis (Arrow 2) in amifostine treatment group (Group 2) (E: H\&E, x40; F: Masson's trichrome staining, $x 40)$

differences were found between groups 1 and 2, groups 1 and 3, and also between groups 1 and 4 in the myocardium of the right ventricles ( $p=0.011, p=0.003, p=0.001$, respectively). Additionally, vasculitis was seen in the right ventricular wall (myocardium and epicardium) in group $1(\mathrm{n}=6)$ and group $2(\mathrm{n}=1)$. It was found significantly higher in group 1 than in group $2(\mathrm{p}=$ $0.027)$, group $3(p=0.014)$ and group $4(p=0.014)$. The number and severity of vasculitis was found significantly diminished in group 2 (Fig. 1E,F) compared to group 1 (Fig. 1C,D). Vasculitis of the epicardial vasculature has a similar severity to myocardial equivalent (Fig. 2C). At the same time, significantly thickened medial layer in association with vascular damage was seen in irradiated groups (groups 1 and 2).

\section{Myocyte necrosis}

Necrosis was seen in groups 1 and 2 (Fig. 2D). There were 5 findings of myocyte necrosis in group 1 [right ventricle, grade $1(n=3)$; left ventricle grade $1(n=3)$, grade $2(n=1)]$ while 3 were detected in group 2 [left ventricle grade $1(n=1)$, grade 2 $(n=1)]$. We did not find any necrosis in non-irradiated groups. The number and severity of necrosis was prominently observed to be high in group 1 in comparison with group 2 . The observational difference did not end up with statistical difference ( $p$ $=0.124)$. 


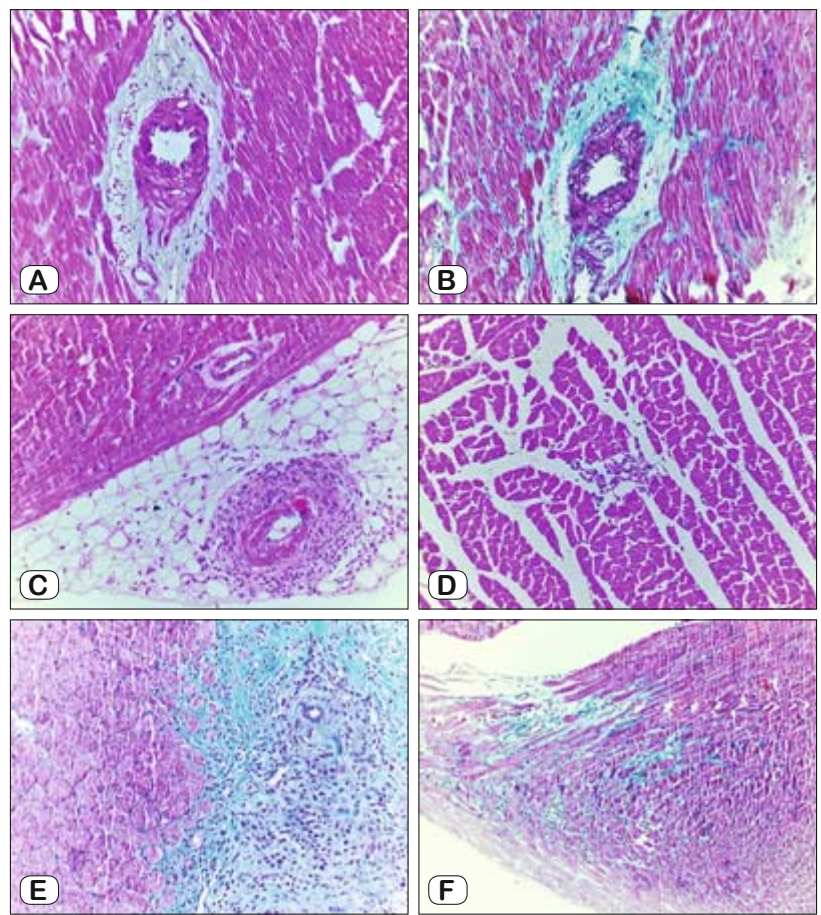

Fig. 2. A: Vasculitis of the epicardial vasculature (H\&E, x200), B: Myosit necrosis characterized by macrophage infiltration (Myophagia) (H\&E, x200), C: Myocardial fibrosis starting from the perivascular area and then progressing into the interstitial field among the myofibrils $(H \& E, x 200)$, D: Dense fibrosis covering each myofibril forming mesh configuration at the apex of the heart (H\&E, x200), E-F: Vascular damage characterized by perivascular fibrosis, thickening of muscular layer and collagen fibers between myofibril (interstitial fibrosis) ( $E$ : H\&E, x200; F: Masson's trichrome staining x200).

\section{Fibrosis}

Myocardial fibrosis was seen particularly in irradiated groups but ameliorative effect of the amifositine could not be found as shown in Table 4. Epicardial fibrosis was found more in the right ventricle in groups 1 and 2 [right ventricle ( $\mathrm{n}=8$ in group $1, \mathrm{n}=$ 7 in group 2), left ventricle ( $\mathrm{n}=1$ in group $1, \mathrm{n}=3$ in group 2)]. We observed myocardial fibrosis that started from the perivascular area and then progressed into the interstitial field among the myofibrils (Fig. 2E). Fibrosis is the final result of the damage afflicted to capillary networks by radiation; therefore, we searched for correlation and found significance between the vascular damage and fibrosis development in the right and left ventricles in group 1 ( $\mathrm{r}$ $=0.715, \mathrm{r}=0.823)$, while a similar correlation was found in left ventricle in group $2(\mathrm{r}=0.821)$

\section{Mesh configuration}

This parameter was present in group 1 with 7 in the right ventricle and 4 in the left ventricle (Fig. 2F). Group 2 had 7 on the right and 6 on the left. The only statistically significant difference was found between groups 2 and $4(p=0.017)$. Mesh configuration was slightly higher in number and severity in group 2 (especially in the left ventricle) when compared to group 1 .

\section{Thrombus}

No thrombus formation was observed in the ventricles of the rats in any of the groups.

\section{Discussion}

In the present study, we have demonstrated that amifostine is able to prevent radiation-induced heart disease, i.e. to reduce vasculitis and vascular injury at 6 months after irradiation. This is the first histopathological report of long-term protection against radiation-induced cardiac damage.

The cytoprotective mechanism of amifostine is complicated, involving free-radical scavenging, DNA protection and repair acceleration, and induction of cellular hypoxia by auto-oxidation (10). Amifostine is believed to be more effective for normal tissues from damage caused by radiation and chemotherapy. The only exception is the central nervous system because amifostine does not cross the blood-brain barrier. Normal tissues have many vessels that contain higher levels of alkaline phosphatase. This enzyme activity is also higher in alkaline $\mathrm{pH}$ than in normal tissues. Several other mechanisms were proposed to explain the selective radioprotection by amifostine, including protection of DNA by metabolites of amifostine, causing hypoxia in normal tissues by increasing oxygen consumption, and accelerated recovery of normal endothelial cells (11).

Radiation causes damage to cells by two mechanisms resulting in direct and indirect effects. If radiation interacts with the atoms of the DNA molecule, or other cellular component critical to the survival of the cell, it is referred to as a direct effect. Indirect effect develops through reactive oxygen species (ROS) that occurs as a result of water radiolysis in cells exposed to ionizing radiation.

Direct effect of RT can be seen in all layers of the heart. The cellular level of RIHD can be explained with oxidative stress which induces endothelial dysfunction and myocardial remodeling characterized by hypertrophy, apoptosis, altered gene expression and increased matrix metalloproteinase activity. Endothelial dysfunction is a primary basic mechanism behind radiation-induced vascular damage. This dysfunction manifests itself by neutrophil chemotaxis, impaired nitric oxide-mediated relaxation (vasodilatation) and ruined adhesions between endothelial cells at early periods of the radiation exposure which we did not study (12).

Certain cytokines and growth factors may stimulate radiationinduced fibroblast proliferation, collagen deposition and fibrosis at the late stages of RT. These changes affect the vessels of each dimension. Damage to the large vessels can result in accelerated atherosclerosis and vasculitis whereas the influence of capillary vessels eventually leads to interstitial fibrosis (pericellular and perivascular) (13).

\section{Chronic inflammation}

Staging of histopathological effects of radiation was published by Fajardo and Stewart. It is described to be as follows: acute stage (6-48 hours), latent stage (2-70 days), late stage ( $>70$ days) (9). In this study, myocardium and epicardium were affected layers from chronic inflammation. Anti-inflammatory effects of 
amifostine following radiation exposure is not clear in literature but some authors demonstrated an anti-inflammatory property of amifostine in acute inflammation (14). Nevertheless, we did not find any amendatory effect on chronic inflammation.

\section{Vascular damage}

Radiation-induced vascular injury is defined by macrovascular injury characterized by accelerated atherosclerosis (coronary artery disease) and microvascular injury manifested by a reduced capillary density causing pericellular and perivascular interstitial fibrosis in myocardium. Another type of vascular injury is vasculitis which is seen mostly in large vessels (15).

We have seen both forms of macrovascular injury. Amifostine pretreatment has significantly reduced vascular damage and vasculitis. This protective effect may be due to the lowered levels of ROS. Taunk et al explained the mechanism of vascular damage and vasculitis by suggesting that the endothelial cells injured by radiation secrete some adhesion molecules and growth factors prompting activation of the inflammatory response and consequently fibrosis. Inflammatory response develops with monocyte chemotactic factor, tumor necrosis factor (TNF), and interleukins (IL), on the other hand platelet-derived growth factor (PDGF), transforming growth factor $b$ (TGF-b), basic fibroblast growth factor (bFGF), insulin like growth factor (IGF), and connective tissue growth factor (CTGF) are profibrotic cytokines (12).

\section{Myocyte necrosis}

Necrosis can occur as a result of microvascular ischemia (15). The existence of myocyte necrosis in long term is probably depending on continuous microvascular damage. Histologically, we observe reduced necrosis in group 2 but this was not showing any statistically significant difference between groups 1 and 2. This result showed that amifostine has a partial potential to reduce myocyte necrosis and microvascular injury.

\section{Fibrosis}

The mechanisms of fibrosis that occurs due to radiation has not yet been fully clarified; however, it is hypothesized that cells are damaged due to radiation beams leading to the breakdown of the capillary networks, thereby causing repeated episodes of ischemia and ultimately fibrosis (15). It is shown in varying degrees of interstitial fibrosis and/or perivascular fibrosis in the ventricle ( 9 , 16, 17). Experimentally, an exposure of $20 \mathrm{~Gy}$ radiation leads to myocardial degeneration and becomes apparent in rats at around 6 months after irradiation and is preceded by a marked reduction in capillary density (18). In this study, variable degrees of ventricular fibrosis were seen in all layers of irradiated groups similar to the findings described in literature.

Epicardium and myocardium are the two layers in which fibrosis has been observed to be most prominent. Radiotherapy group (group 1) manifested all grades of fibrosis in both sides of ventricles.

Fibrillar collagen appears after cardiocyte necrosis in order to replace the lost cells (19). It is reported that perivascular fibrosis of intramural arterioles, thickening of preexisting collagen fibers and an increase in the number of fibers could be seen between viable myocytes after the radiation exposure (20). We saw the same observation similar to our previous study (21), namely that myocardial fibrosis started from the perivascular area and then progressed into the interstitial field among the myofibrils. In the set of mesh configuration, severe myocardial fibrosis was seen at apex of the heart; tip of papillary muscles and in areas where the ventricle wall was adjacent to the atrioventricular valves.

The literature does not clearly denote that amifositine treatment reduces fibrosis but Kruse et al showed lower fibrosis scores for atrial appendages, left ventricles and perivascular fibrosis in animals treated with amifostine prior to irradiation when compared to those with the same irradiation dose (7). In contrary to this, we found that amifostine treatment did not decrease myocardial fibrosis, and our conclusion is that it was far from being effective in protecting from myocardial fibrosis.

\section{Mesh configuration}

Mesh, which is a form of fibrosis, stands out when the severity of fibrosis is increased. Mesh was found in study groups as expected but we could not observe beneficial effects of preventing the mesh. The development of fibrosis is a process form of cardiomyopathy and valvular heart diseases that form the subgroup of RIHD. Furthermore, valve regurgitation and/or stenosis caused by fibrosis have been reported in literature (22). In the present study we have not demonstrated that amifostine is able to prevent radiation-induced reduction in terms of fibrosis occurrence at 6 months after irradiation.

\section{Thrombus formation}

Intraventricular and/or intravascular thrombus was not identified. Radiation may not be a direct thrombogenic effect or thrombus may have occurred and dissolved in the early stage.

\section{Conclusion}

Elementary pathological lesions of RIHD are fibrosis, vascular damage, vasculitis and myocyte necrosis in the heart. Myocardium and epicardium were the mostly affected layers of the heart.

The development of vasculitis and vascular injury was prevented by amifostine treatment. Fibrosis formation of myocardial and epicardial layers were not decreased by the use of amifositine treatment. The number and severity of myocyte necrosis were observed to be diminished by the amifositine treatment despite the statistically non-significant result. The anti-inflammatory effect of amifositine was not found in chronic inflammation.

Prevention of RIHD with the use of amifositine is encouraging based on histopathological results that we brought forward in this study.

\section{References}

1. Fuster V, Voute J. MDGs: chronic diseases are not on the agenda. Lancet 2005 ; 366: 1512-1514. 
2. Stewart FA. Mechanisms and dose-response relationships for radiationinduced cardiovascular disease. Ann ICRP 2012; 41 (3-4): 72-79.

3. Prosnitz RG, Marks LB. Radiation-induced heart disease: vigilance is still required. J Clin Oncol 2005; 23 (30): 7391-7394.

4. Cannel RJ, Kaplan HS. Mantle irradiation in Hodgkin's disease:an analysis of technique, tumor eradication, and complications. Cancer 1976; 37: $2813-2825$.

5. Alberts DS, Speicher LA, Krutzsch M et al. WR-1065, the active metabolite of amifostine (Ethyol), does not inhibitthe cytotoxic effects of a broad range of standard anticancer drugs against human ovarian and breast cancer cells. Eur J Cancer 1996; 32: 17-20.

6. Kamran MZ, Ranjan A, Kaur N, Sur S, Tandon V. Radioprotective Agents:Strategies and Translational Advances. Med Res Rev 2016; 36 (3): 461-493.

7. Kruse JJ, Strootman EG, Wondergem J. Effects of amifostine on radiation-induced cardiac damage. Acta Oncol 2003; 42 (1): 4-9.

8. Tokatli F, Uzal C, Doganay L et al. The potential cardioprotective effects of amifostine in irradiated rats. Int J Radiat Oncol Biol Phys 2004; 58 (4): 1228-1234.

9. Fajardo LF, Stewart JR. Experimental radiation-induced heart disease. I. Light microscopic studies. Am J Pathol 1970; 59 (2): 299-316.

10. Giambarresi L, Jacobs AJ. Radioprotectants. In: Conklin JJ, Walker RI, editors. Military Radiobiology. Orlando, FL: Academic Press, 1987, 265-301.

11. Capizzi RL. Amifostine: the preclinical basis for broad spectrum selective cytoprotection of normal tissues from cytotoxic therapies. Semin Oncol 1996; 23: 2-17.

12. Taunk NK, Haffty BG, Kostis JB, Goyal S. Radiation-induced heart disease: pathologic abnormalities and putative mechanisms. Front Oncol 2015; 39 (5): $1-8$.
13. Korpela E, Liu SK. Endothelial perturbations and therapeutic strategies in normal tissue radiation damage. Radiat Oncol 2014; 266 (8): 1-9.

14. Bhutia YD, Vijayaraghavan R, Pathak U. Analgesic and anti-inflammatory activity of amifostine, DRDE-07, and their analogs, in mice. Indian J Pharmacol 2010; 42 (1): 17-20.

15. Darby SC, Cutter DJ, Boerma M. Radiation-related heart disease: current knowledge and future prospects. Int J Radiat Oncol Biol Phys 2010; 76 (3): 656-665.

16. Stewart JR, Fajardo LF, Gillette SM. Radiation injury to the heart. Int J Radiat Oncol Biol Phys 1995; 31: 1205-1211.

17. Schultz-Hector S, Bohm M, Blochel A. Radiation-induced heart disease:morphology, changes in catecholamine synthesis and content, beta-adrenoceptor density, and hemodynamic function in an experimental model. Radiat Res 1992; 129: 281-289.

18. Schultz-Hector S, Balz K. Radiation-induced loss of endothelial alkaline phosphatease activity and development of myocardial degeneration. An ultrastructural study. Lab Invest 1994; 71: 252-260.

19. Skalli O, Schurch W, Seemayer T. Myofibroblasts from diverse pathologic settings in their content of actin isoforms and intermediate filament proteins. Lab Invest 1989; 60: 275-285.

20. Weber KT, Sun Y, Katwa LC. Connective tissue and repair in the heart. Potential regulatory mechanisms. Ann NY Acad Sci 1995; 27 (752): 286-299.

21. Gürses I, Özeren M, Serin M, Yücel N, Erkal HŞ. Histopathological evaluation of melatonin as a protective agent in heart injury induced by radiation in a rat model. Pathol Res Pract 2014; 210 (12): 863-871.

22. Filopei J, Frishman W. Radiation-induced heart disease. Cardiol Rev 2012; 20: 184-188.

Received August 25, 2017. Accepted October 16, 2017. 